\title{
A pilot program to introduce Interprofessional Education in a Dental Pharmacology Course
}

\author{
Jayne Reuben ${ }^{1}$, Kathleen Eddy ${ }^{2}$, Lyndsay Cole ${ }^{2}$, Shaun Logan ${ }^{1}$, Kiara Talabi $^{2}$ \\ ${ }^{1}$ Biomedical Sciences, Texas A \& M University College of Dentistry, USA, ${ }^{2}$ Baylor Scott and White Health, USA
}

Background: Medication errors remain one of the most preventable clinical errors that can lead to patient harm. An increasing number of reports suggest that these errors can be minimized when health professionals work collaboratively and utilize the skill sets of each team member to patient care. As a result, interprofessional activities have been added into the curricula for medical, nursing, dental and pharmacy schools. However, this presents a challenge for the Texas A \& M University (TAMU) College of Dentistry which is not physically close to other health profession programs within the TAMU system. To address this problem, resident pharmacists from the Baylor Scott and White Community Care Clinics were invited to present the legal and practical aspects of prescription writing to second-year dental students. The purpose of this study was to assess student attitudes regarding this interprofessional encounter.

Methods: Two first-year pharmacy residents presented information on state and federal drug laws. In addition, students received instruction and practice on accurate prescription writing. Dental students were given this survey before and after the pharmacy resident presentations. One hundred second-year dental students were enrolled in a dental pharmacology course of which 68 students completed a survey at two time points. Survey questions were divided into three categories: student attitudes concerning the information presented, the value of interprofessional patient care and the role of pharmacists as health care providers. Descriptive statistics were computed to provide a summary of the responses.

Results: The majority of the students surveyed (96\%) agreed that the sessions provided relevant knowledge for their practice of dentistry and that presentations from other health professionals were valuable (95\%). After the presentations, there number of students who recognized that pharmacists have multiple roles in patient care increased by forty-five percent (45\%).

Conclusion: In addition to learning about prescription writing, exposure to pharmacy health professionals in a dental training program appeared to lead to a greater appreciation of the roles, responsibilities and contributions of the pharmacist as a member of the health care team. These encounters may increase the likelihood of dental students engaging pharmacists during their training and after graduation. 